\title{
Measuring vincristine-induced peripheral neuropathy in children with cancer: validation of the Dutch pediatric-modified Total Neuropathy Score
}

\author{
S. M. Schouten ${ }^{1} \cdot$ M. E. van de Velde ${ }^{1}$ (D) - G. J. L. Kaspers ${ }^{1,2} \cdot$ L. B. Mokkink ${ }^{3} \cdot$ I. M. van der Sluis ${ }^{2} \cdot$ C. van den Bos $^{2,4}$. \\ A. Hartman ${ }^{5}$. F. C. H. Abbink ${ }^{4}$ - M. H. van den Berg ${ }^{1}$
}

Received: 21 February 2019 / Accepted: 30 September 2019/Published online: 16 November 2019

(C) The Author(s) 2019

\begin{abstract}
Purpose The aims were to evaluate the construct validity and reliability of the Dutch version of the pediatric-modified Total Neuropathy Score (ped-mTNS) for assessing vincristine-induced peripheral neuropathy (VIPN) in Dutch pediatric oncology patients aged 5-18 years.

Methods Construct validity (primary aim) of the ped-mTNS was determined by testing hypotheses about expected correlation between scores of the ped-mTNS (range: 0-32) and the Common Terminology Criteria for Adverse Events (CTCAE) (range: 018) for patients and healthy controls and by comparing patients and controls regarding their total ped-mTNS scores and the proportion of children identified with VIPN. Inter-rater and intra-rater reliability and measurement error (secondary aims) were assessed in a subgroup of study participants.

Results Among the 112 children (56 patients and 56 age- and gender-matched healthy controls) evaluated, correlation between CTCAE and ped-mTNS scores was as expected (moderate $(r=0.60)$ ). Moreover, as expected, patients had significantly higher ped-mTNS scores and more frequent symptoms of VIPN compared with controls (both $p<.001$ ). Reliability as measured within the intra-rater group $(n=10)$ (intra-class correlation coefficient $\left(\mathrm{ICC}_{\text {agreement }}\right)=0.64$, standard error of measurement $\left(\mathrm{SEM}_{\text {agreement }}\right)=2.92$, and smallest detectable change $\left.\left(\mathrm{SDC}_{\text {agreement }}\right)=8.1\right)$ and within the inter-rater subgroup $(n=10)$ $\left(\mathrm{ICC}_{\text {agreement }}=0.63, \mathrm{SEM}_{\text {agreement }}=3.7\right.$, and $\left.\mathrm{SDC}_{\text {agreement }}=10.26\right)$ indicates insufficient reliability.

Conclusion The Dutch version of the ped-mTNS appears to have good construct validity for assessing VIPN in a Dutch pediatric oncology population, whereas reliability appears to be insufficient and measurement error high. To improve standardization of VIPN assessment in children, future research aimed at evaluating and further optimizing the psychometric characteristics of the ped-mTNS is needed.
\end{abstract}

Keywords Neurotoxicity $\cdot$ Diagnostics $\cdot$ Pediatrics $\cdot$ Cancer $\cdot$ Vincristine $\cdot$ Toxicity

S. M. Schouten and M. E. van de Velde contributed equally to this work.

Electronic supplementary material The online version of this article (https://doi.org/10.1007/s00520-019-05106-3) contains supplementary material, which is available to authorized users.

M. E. van de Velde

mi.vandevelde@amsterdamumc.nl

1 Emma Children's Hospital, Amsterdam UMC, Vrije Universiteit Amsterdam, Pediatric Oncology Amsterdam,

Amsterdam, The Netherlands

2 Princess Máxima Center for Pediatric Oncology, Utrecht, The Netherlands
3 Department of Epidemiology and Biostatistics and Amsterdam Public Health Research Institute, VU University Medical Center, Amsterdam, The Netherlands

4 Emma Children's Hospital, Amsterdam UMC, Amsterdam Medical Center, Pediatric Oncology Amsterdam, Amsterdam, The Netherlands

5 Department of Pediatric Physiotherapy, Erasmus Medical Center Rotterdam/Sophia Children's Hospital, Rotterdam, The Netherlands 


\section{Introduction}

Vincristine (VCR) is a chemotherapeutic agent which is often used in pediatric oncology for the treatment of various hematological and solid cancers [1]. A frequently occurring side-effect of VCR is peripheral neuropathy [2]. VCR-induced peripheral neuropathy (VIPN) is a mixed sensory, motor and autonomous neuropathy mainly affecting the longer peripheral nerves. Symptoms of VIPN usually start in the distal part of the limbs and may progress proximally [3]. Symptoms include paresthesia, numbness, tingling, loss of proprioception and pain. Rarely, VIPN may lead to profound muscle weakness with symptoms as foot drop and walking difficulties. Autonomous symptoms of VIPN include constipation and dizziness [4]. VIPN showed to adversely affect quality of life in oncology patients [5].

VIPN is a multifactorial toxicity, which is influenced by several determinants. Older children seem to be more affected than younger children, whereas the influence of gender on VIPN is unclear [6]. Moreover, there is a racial difference: Caucasian children tend to have more VIPN than nonCaucasian children [7]. Furthermore, although there is a dose dependent relation between VCR and [8], studies assessing associations between pharmacokinetic parameters of VCR and VIPN report conflicting results $[9,10]$. Finally, genetic factors are known to influence susceptibility to VIPN, with several pathways involved such as CEP72 and CYP3A4/5 [8, $11]$.

Treatment of VIPN is mainly symptomatic using analgesics such as gabapentin, or laxatives in case of constipation. Another treatment option is dose reduction of VCR, although this may lead to suboptimal treatment of the underlying malignancy [12].

In clinical practice, several tools are used to measure VIPN in pediatric oncology patients. The Common Terminology Criteria for Adverse Events (CTCAE) is a tool that assesses the severity of several types of adverse events in oncology patients [13], including those regarding peripheral neuropathy, constipation and neuralgia. Furthermore, Lavoie-Smith et al. [14, 15] developed the Total Neuropathy Score-Pediatric Vincristine (TNS-PV) specifically for the assessment of VIPN in pediatric oncology patients. The TNS-PV consists of a seven-item interview-based questionnaire and a standardized physical examination (testing of vibration and temperature sense, muscle strength and deep tendon reflexes (DTR)) [6]. Besides questionnaires and/or physical examinations, nerve conduction studies in which the conduction velocity of different nerves is measured using somatosensory evoked potentials $[16,17]$ can also be used to assess VIPN.

However, all of the above-mentioned methods to assess VIPN have some limitations. Although frequently used, the CTCAE shows insufficient sensitivity in detecting motor and sensory neuropathy [2]. Moreover, the TNS-PV can only be used in children aged 6 years or older [14]. Furthermore, nerve conduction studies are invasive, painful, and expensive [14]. Finally, physical examination can only validly be performed and interpreted by specifically trained physicians such as pediatric neurologists $[18,19]$.

Recently, the pediatric-modified Total Neuropathy Score (ped-mTNS) was developed [20]. This instrument, which consists of an interview-based questionnaire and physical examination, showed to have superior psychometric characteristics compared with other tools for the assessment of peripheral neuropathy in children [2]. Moreover, it is a quick, inexpensive, and non-invasive tool that can be employed by different health care professionals, such as physicians, physical therapists, and nurses [4]. However, the psychometric characteristics of the pedmTNS have solely been evaluated in North American children with cancer aged 5-18 years.

The primary aim of the current study was to evaluate the construct validity of the Dutch version of the ped-mTNS for the assessment of VIPN in Dutch pediatric oncology patients aged 5-18 years. In addition as secondary aim, reliability of this tool was investigated in a subpopulation.

\section{Methods}

\section{Study population}

The study population of this multicenter cross-sectional study consisted of pediatric oncology patients aged 5-18 years with non- non central nervous system (CNS) malignancies and healthy controls.

Patients were eligible for study participation if they were treated with at least four administrations of at least $1.5 \mathrm{mg} / \mathrm{m}^{2}$ (maximum $2 \mathrm{mg}$ ) VCR within a period of 6 weeks during treatment of their current malignancy. VIPN assessments were performed within the time frame of at least one month after start of VCR therapy and two months after cessation of VCR therapy maximally. This resulted in the inclusion of patients with the following diagnoses and treatment protocols for the current study: acute lymphoblastic leukemia (ALL) (DCOG ALL-11 protocol [21]), Hodgkin lymphoma (EuroNetPHL-C1 protocol [22] or C2 protocol [23]), nephroblastoma (SIOP Wilms 2001 protocol [24]), and rhabdomyosarcoma (EpSSG RMS 2005 protocol [25]). VCR was administered either by means of a bolus injection (1-5 min) or a 1-h infusion. The healthy control group consisted of siblings of participating patients and children known to hospital co-workers (relatives or friends). Controls were age- $( \pm 1$ year $)$ and gendermatched to the patients on a 1:1 basis. Patients and 
controls were excluded in case of premorbid developmental disorders, neuromuscular disorders, lower extremity amputations, diabetes mellitus, or in case they were not able to speak or understand the Dutch language. Although strictly speaking the included controls could not develop VIPN (as they did not receive any VCR), hypothetically they may have some degree of peripheral neuropathy. The ped-mTNS is a tool which assesses peripheral neuropathy, either chemotherapyinduced or not, in children. For reasons of clarity, we use the term VIPN in the remainder of this paper, thereby referring to either VCR-induced (patients) or nonVCR-induced (controls) peripheral neuropathy.

\section{ped-mTNS}

The original, English version of the ped-mTNS was translated into Dutch by a non-native English speaker, followed by a translation back to English by a bilingual DutchEnglish speaker. Subsequently, this back-translated version was sent to the principal investigator of the original ped-mTNS in the USA [4] in order to have it reviewed and checked by its original developer. Appendix A and B as Supplementary Materials contain the original version and the Dutch version of the ped-mTNS as approved by the developer, respectively.

The questionnaire-part of the ped-mTNS contains eight questions about sensory, functional and autonomic symptoms. These questions were read out aloud by the assessor to the participant. In addition, five different aspects of VIPN were assessed by physical examination: light-touch sensation by Semmes-Weinstein monofilaments (RolyanAbility One, Germantown, WI, USA) [4, 26], pin sensibility by Medipin ${ }^{\mathrm{TM}}$ (Ltd, Hertfordshire, UK) [4], muscle strength by means of manual muscle testing (graded according to the Medical Research Council guidelines) [4, 27], and DTR of the Achilles and patella (graded according to the Mayo Clinic Criteria) $[4,28]$. Due to the unavailability of a Biothesiometer ${ }^{\mathrm{TM}}$ (Biomedical Instruments, Newbury, OH, USA) in The Netherlands, the assessment of vibration sense was carried out with a Rydel-Seiffer 64$\mathrm{Hz}$ tuning fork (Gebrueder Martin, Tuttlingen, Germany), which showed to be a valid instrument in the assessment of vibration sense in children [4, 29]. For all items in the questionnaire and the physical examination part, the score ranged between 0 (no symptoms) and 4 (severe symptoms). The worst scores within each of the 3 items as assessed by the questionnaire, together with the scores of the 5 items tested in the physical examinations, are used to calculate the total ped-mTNS score, which is the sum of these 8 scores (range 0 to 32 ). Children with a total ped-mTNS score of 5 or higher were considered to have VIPN [4].

\section{CTCAE}

The CTCAE (version 4.03) [13] consists of over 330 items scoring adverse events due to cancer treatment divided into 26 different categories. Possible grades range from 0 (no symptoms) and 3 (severe symptoms) or, if the adverse event can be deadly, category 4 is life threatening and category 5 is death. The CTCAE items used for the assessment of VIPN are constipation, peripheral sensory neuropathy, peripheral motor neuropathy, and neuralgia. The maximum CTCAE sum score of these 4 items is 18. Participants with a total score of 2 or higher are considered to have VIPN [6]. The assessor asked to which extent the participants experienced problems as described in the relevant CTCAE items. For the item peripheral sensory neuropathy, additionally DTR of the patella and Achilles were assessed.

\section{Procedures}

Patients were included in Emma Children's Hospital/ Amsterdam University Medical Centers and Sophia Children's Hospital/Erasmus Medical Center Rotterdam. Study measurements were performed between September 2016 and July 2017. All study participants (patients and controls) were tested once using both the ped-mTNS and CTCAE to assess VIPN. These measurements were performed by the same assessor (SS), who was trained extensively by a pediatric neurologist to perform the VIPN assessments. Furthermore, a subset of randomly selected patients and healthy controls were assessed twice in order to assess intra-rater ( 5 patients and 5 controls), both measurements performed by the same assessor) and inter-rater reliability (( 7 patients and 3 controls) second measurement performed by a different assessor $(\mathrm{MvdV})$, who was also specifically trained for the study measurements). All patients were measured during regular hospital visits. Healthy controls were measured either during family visits in the hospital or at home. For the assessment of intra-rater reliability, an interval of 4-16 days between the two measurements, without VCR administrations in between, was adhered to.

\section{Statistics}

Assessment of construct validity was determined by calculating the correlation between the ped-mTNS and the CTCAE sum scores in patients as well as the actual differences between these scores. We hypothesized total ped-mTNS and total CTCAE scores to be moderately correlated, since some items between the two systems attempt to measure the same items (peripheral motor neuropathy and peripheral sensory neuropathy), whereas some items are only present in one of the instruments (constipation is only an item in the CTCAE and not in the ped-mTNS). Moreover, we expected to assess a higher correlation between the two measurement tools than 
the correlation measured in the study of Gilchrist et al. $(r=0.07)$ [2], since in that study the CTCAE scores were retrieved from medical records instead of the prospective assessment of CTCAE scores used in our study. Either the Spearman or Pearson correlation coefficients were calculated, depending on normality of data distribution. According to Nunnally and Bernstein [30], an ICC of $>0.70$ was considered as good. Furthermore, a correlation of $0.51-0.69$ was considered moderate and $<0.5$ as low [31]. We hypothesized ped-mTNS scores of patients to be significantly higher compared with healthy controls and the proportion of patients with VIPN to be significantly higher than those of healthy controls. Differences between mean total ped-mTNS score of patients and controls were analyzed using a Mann-Whitney $U$ test. Differences between the proportion of patients and controls who were identified as having VIPN by the ped-mTNS were calculated using a Chisquare test. A two-tailed significance level of 0.05 was used.

Assessment of reliability and standard error of measurement of the ped-mTNS (secondary aims) was carried out as follows. Reliability and measurement error were measured within the inter-rater and intra-rater subgroups by means of intra-class correlation coefficient using the two-way random effects model for agreement $\left(\mathrm{ICC}_{\text {agreement }}\right)$ according to the equation $\mathrm{ICC}_{\text {agreement }}$ $=\frac{\sigma_{p}^{2}}{\sigma_{p}^{2}+\sigma_{o}^{2}+\sigma_{\text {residal }}{ }^{2}}$ where $\sigma_{\text {error }}{ }^{2}=\sigma_{o}{ }^{2}+\sigma_{\text {residual }}{ }^{2}$ [32]. Measurement error was assessed by calculating the standard error of measurement from the same model (i.e., the two-way random effects model for agreement $\left.\left(\mathrm{SEM}_{\text {agreement }}\right)\right)$ by the equation $\mathrm{SEM}_{\text {agreement }}=\sqrt{\left(\sigma_{o}^{2}+\sigma_{\text {residual }}{ }^{2}\right)}$ and by calculating the smallest detectable change ( $\left.\mathrm{SDC}_{\text {agreement }}\right)$ using the equation $\mathrm{SDC}_{\text {agreement }}=1.96 \times \sqrt{2} \times \mathrm{SEM}_{\text {agreement }}[33,34]$.

Dichotomized total scores of the ped-mTNS (yes/no VIPN; a total ped-mTNS score of 0-4 indicates no VIPN and a score of $\geq 5$ indicates VIPN according to published data by Gilchrist et al. [4]) were used to measure agreement in a $2 \times 2$ table to calculate positive agreement $(\mathrm{PA})(\mathrm{PA}=2 a /(2 a+b+c)$ and negative agreement (NA) (NA $=2 d /(2 d+b+c)$.

All results on reliability are reported for patients, healthy controls, and total group separately. All statistics were performed using SPSS for Windows version 22.0 (SPSS, Chicago, IL).

\section{Results}

\section{Study population}

Fifty-six patients and 56 healthy controls (median, interquartile range (IQR) age 9.6 (6.6-14.2) years) were included in the study. Characteristics of the participants are presented in Table 1 . The majority of the patients were treated for ALL $(n=39,70 \%)$ or Hodgkin lymphoma $(n=12,21 \%)$. The mean (standard deviation (SD)) cumulative dose of VCR administered to the patients was $19.6 \mathrm{mg} / \mathrm{m}^{2}$ (13.9).
Table 1 Baseline characteristics of study participants

\begin{tabular}{|c|c|c|}
\hline & $\begin{array}{l}\text { Patients } \\
(n=56)^{1}\end{array}$ & $\begin{array}{l}\text { Healthy controls } \\
(n=56)^{1}\end{array}$ \\
\hline \multicolumn{3}{|l|}{ Sex } \\
\hline Male & $32(57.1)$ & $32(57.1)$ \\
\hline Female & $24(42.9)$ & $24(42.9)$ \\
\hline Age in years (median [IQR]) & $9.7[6.3-14.1]$ & $9.6[7.0-14.4]$ \\
\hline \multicolumn{3}{|l|}{ Ethnicity } \\
\hline European & $40(71.4)$ & $39(69.6)$ \\
\hline Middle-Eastern & $10(17.9)$ & $6(10.7)$ \\
\hline African & $1(1.8)$ & $4(7.1)$ \\
\hline Hispanic & & $4(7.1)$ \\
\hline Other & $5(8.9)$ & $3(5.4)$ \\
\hline \multicolumn{3}{|l|}{ Diagnosis $^{2}$} \\
\hline ALL & $39(69.6)$ & \\
\hline Hodgkin lymphoma & $12(21.4)$ & \\
\hline Nephroblastoma & $1(1.8)$ & \\
\hline Rhabdomyosarcoma & $4(7.1)$ & \\
\hline \multicolumn{3}{|l|}{ Administration method of $\mathrm{VCR}^{2}$} \\
\hline Bolus injection & $47(83.9)$ & \\
\hline 1-h infusion & $9(16.1)$ & \\
\hline $\begin{array}{l}\text { Cumulative dose of VCR } \\
\left(\mathrm{mg} / \mathrm{m}^{2} ; \text { mean }(\mathrm{SD})\right)^{2}\end{array}$ & $19.6(13.91)$ & \\
\hline $\begin{array}{l}\text { Time since last VCR do se (days, } \\
\text { median }[\mathrm{IQR}])^{2}\end{array}$ & $9.5[0.75-21.0]$ & \\
\hline
\end{tabular}

${ }^{1}$ Values represent the number $(\%)$ of participants, unless indicated otherwise; ${ }^{2}$ This is only applicable to the patient population

$I Q R$ interquartile range, $A L L$ acute lymphoblastic leukemia, $V C R$ vincristine, $S D$ : standard deviation

\section{Construct validity of the ped-mTNS}

The correlation between total scores of the ped-mTNS and CTCAE was moderate as expected in patients $(r=0.60)$. Patients had a significantly higher score on ped-mTNS than healthy controls (median (IQR): $10.0(6.25-13.0)$ and median (IQR): $0.0(0.0-1.0)$, respectively; $p<0.001)$ (Table 2$)$. Furthermore, patients were significantly more often identified as having VIPN ( $\geq 5$ on the ped-mTNS) than healthy controls ( $86 \%$ versus $1.8 \%$, respectively; $p<0.001$ ).

\section{Reliability}

All results regarding reliability for patients, healthy controls, and the total group of participants are depicted in Table 3 . The different variance components of the intra-rater reliability group were: $\sigma_{\mathrm{p}}{ }^{2}=15.3, \sigma_{\mathrm{o}}^{2}=0$, and $\sigma_{\text {residual }}{ }^{2}=8.6$ and those of the inter-rater reliability were: ${\sigma_{\mathrm{p}}}^{2}=23.1,{\sigma_{\mathrm{o}}}^{2}=0$, and $\sigma_{\text {residual }}{ }^{2}=13.7$. The $\mathrm{ICC}_{\text {agreement }}$ of the intra-rater group was 0.64 , whereas this was 0.63 of the inter-rater group. Positive 
Table 2 Results of peripheral neuropathy measurements in the two study groups

\begin{tabular}{llll}
\hline & $\begin{array}{l}\text { Patients } \\
(n=56)\end{array}$ & $\begin{array}{l}\text { Healthy controls } \\
(n=56)\end{array}$ & $p$ value \\
\hline $\begin{array}{l}\text { Total ped-mTNS scores } \\
\text { Median [IQR }]\end{array}$ & $10.0[6.25-13.0]$ & $0.0[0.0-1.0]$ & $<0.001$ \\
Score of 0-4 $(n,(\%))$ & $8(14.3)$ & $55(98.2)$ & \\
Score 5-32 $(n,(\%))$ & $48(85.7)$ & $1(1.8)$ & \\
Total CTCAE scores & & & $<0.001$ \\
Median [IQR] & $3[2-4.75]$ & $0[0-0]$ & \\
Score of 0-1 $(n,(\%))$ & $9(16.1)$ & $55(98.2)$ & \\
Score of 2-4 $(n,(\%))$ & $33(59.0)$ & $1(1.8)$ & \\
Score of 5-7 $(n,(\%))$ & $12(21.4)$ & $0(0)$ & \\
Score of $8-18(n,(\%))$ & $2(3.6)$ & $0(0)$ & \\
\hline
\end{tabular}

ped-mTNS pediatric-modified Total Neuropathy Score, IQR interquartile range CTCAE Common Terminology Criteria for Adverse Events

agreement was $80 \%$, negative agreement was $83 \%$. Positive agreement and negative agreement of inter-rater reliability were both $100 \%$. Finally, the $\mathrm{SEM}_{\text {agreement }}$ within the intrarater group was 2.92 and within the inter-rater group 3.7. The $\mathrm{SDC}_{\text {agreement }}$ was 8.1 within the intra-rater group and 10.26 within the inter-rater group.

\section{Discussion}

In this study, the Dutch version of the ped-mTNS was validated by assessing VIPN in a cohort of Dutch pediatric oncology patients aged 5-18 and age- and gender-matched healthy controls. The correlation between ped-mTNS and CTCAE was found to be as expected (i.e., moderate). Furthermore, patients had significantly higher ped-mTNS scores than controls and were significantly more often identified as having VIPN. These results indicate that this translated version of the pedmTNS has good construct validity regarding the assessment of VIPN in Dutch pediatric oncology patients. However, reliability of this instrument was insufficient $\left(\mathrm{ICC}_{\text {agreement }}<0.7\right)$. The outcomes of measurement error showed a SEM agreement of 2.92 and 3.7 and $\mathrm{SDC}_{\text {agreement }}$ of 8.1 and 10.26 for the intrarater and inter-rater subgroups, respectively. Although the minimal important change (MIC) of this instrument is unknown, these $\mathrm{SEM}_{\text {agreement }}$ and $\mathrm{SDC}_{\text {agreement }}$ Scores appear to be rather high, given the fact that scores can range from 0 to 32 and we used a cutoff value of $\geq 5$ to discriminate children with and without VIPN. However, positive agreement and negative agreement were good, with scores between 80 and $100 \%$ for intra-rater and inter-rater reliability, respectively.

The results of the current study are comparable to a previous study. Gilchrist et al. [4] recently showed that patients had a significantly higher score on ped-mTNS than healthy controls (mean (SD): 8.7 (4.2); and 1.4 (0.9), respectively). However, in that study, only $9.8 \%$ of the controls had a score of 0 on the ped-mTNS, while this percentage was $68 \%$ in the current study. This discrepancy can most likely be attributed to differences in carrying out the assessments with the pedmTNS, such as the level of training of the assessors, and not to a difference in population. The results of Gilchrist et al. showed that healthy controls frequently experienced some disorders of motor function, autonomic symptoms, pin sensation, and distal strength, although in both studies scores of healthy individuals were not high enough to indicate VIPN (ped-mTNS score was $<5$ ) and therefore not of great clinical relevance. In the current study, the healthy control group had a mean (SD) ped-mTNS score of 0.9 (1.5). In the study of Gilchrist et al., similar scores were reported (mean (SD): 1.4 (0.9)). Furthermore, the current study showed an $\mathrm{ICC}_{\text {agreement }}$ score of the ped-mTNS for intra- and inter-rater reliability of 0.64 and 0.63 , respectively. These scores are lower than the ICC sores for intra-rater and inter-rater reliability as reported by Gilchrist et al. (0.99 and 0.98, respectively) [4]. This may be due to the fact that within the study of Gilchrist et al., an interval of only $1 \mathrm{~h}$ between two measurements was applied

Table 3 Reliability outcome measures of the pediatric-modified Total Neuropathy Score

\begin{tabular}{|c|c|c|c|c|c|c|}
\hline & $\begin{array}{l}\mathrm{ICC}_{\text {agreement }} \text { total } \\
\text { ped-mTNS score }\end{array}$ & $\begin{array}{l}95 \% \text { CI ICC total } \\
\text { ped-mTNS score }\end{array}$ & $\mathrm{SDC}_{\text {agreement }}$ & SEM $_{\text {agreement }}$ & $\begin{array}{l}\text { Positive agreement } \\
\text { of total ped-mTNS } \\
\text { score }\end{array}$ & $\begin{array}{l}\text { Negative agreement } \\
\text { of total ped-mTNS } \\
\text { score }\end{array}$ \\
\hline Inter-rater reliability patients $(n=7)$ & 0.45 & -0.43 to 0.88 & 12.25 & 4.42 & $100 \%$ & $100 \%$ \\
\hline $\begin{array}{l}\text { Inter-rater reliability healthy } \\
\text { controls }(n=3)\end{array}$ & 0 & - & 5.73 & 2.07 & - & $100 \%$ \\
\hline Intra-rater reliability patients $(n=5)$ & 0 & -1.3 to 0.20 & 8.96 & 3.23 & $80 \%$ & $0 \%$ \\
\hline $\begin{array}{l}\text { Intra-rater reliability healthy } \\
\text { controls }(n=5)\end{array}$ & 0 & - & 0 & 0 & - & $100 \%$ \\
\hline Inter-rater reliability total $(n=10)$ & 0.63 & 0.04 to 0.89 & 10.26 & 3.7 & $100 \%$ & $100 \%$ \\
\hline Intra-rater reliability total $(n=10)$ & 0.64 & 0.06 to 0.90 & 8.1 & 2.92 & $80 \%$ & $83 \%$ \\
\hline
\end{tabular}

ICC intra-class correlation coefficient, ped-mTNS pediatric-modified Total Neuropathy Score, $C I$ confidence interval, $S D C$ smallest detectable change, SEM standard error of measurement 
when assessing inter- and intra-rater reliability, whereas in our study, this interval was 4 days minimally. An interval of $1 \mathrm{~h}$ may have led to recall bias of the patient for the interviewed questions and of the assessor for the physical examination part, since there is a high chance that the results of the previous measurement are memorized. Furthermore, Gilchrist et al. have used a two-way mixed effect model for consistency for their calculation of ICC (i.e. $\operatorname{ICC}_{\text {consistency }}$ ); therefore, the variance due to systematic differences between observers was ignored [32, 33]. By using $\mathrm{ICC}_{\text {agreement, }}$ which we did in our study, this variance of observers was taken into account. However, using $\mathrm{ICC}_{\text {consistency }}$ instead of $\mathrm{ICC}_{\text {agreement }}$ leads to higher ICC values, which could be an explanation for the difference in results between these studies [35].

Our study was the first to evaluate psychometric characteristics of a translated version of the ped-mTNS. Our results are important both for clinical purposes and research practice as they contribute to the urgently needed standardization of measuring peripheral neuropathy in children with cancer using high-quality outcome measurement instruments.

This study has some limitations. As previously mentioned, the Rydel-Seiffer $64-\mathrm{Hz}$ tuning fork was used for the assessment of vibration sense instead of the Biothesiometer ${ }^{\mathrm{TM}}$, due to unavailability of this device in The Netherlands. However, according to Hilz et al. [29], the Rydel-Seiffer 64-Hz tuning fork is a valid instrument to examine vibration sense and Gilchrist et al. [20] showed a moderate to good correlation (i.e., $r=-$ 0.62 to -0.73 , depending on test site) between the BiothesiometerTM and the Rydel-Seiffer 64-Hz tuning fork.

The assessment of reliability and measurement error were only evaluated within a subgroup of 20 participants. Although there is no formal consensus about the minimal number of participants needed to properly asses the inter- and intrarater reliability [36], the number of participants included for these assessments is probably rather low as can be seen in the 95\% confidence intervals of the reliability estimations, due to logistical limitations. Therefore, these results should be interpreted with caution. Future studies assessing the reliability of the ped-mTNS in a larger group of patient are advocated. These studies can be performed within a patient population, since results regarding reliability of healthy controls will probably be difficult to interpret due to floor effects.

Despite certain psychometric limitations, the ped-mTNS is currently considered to be the most optimal instrument for assessing VIPN in children compared with other instruments [37]. Continuous efforts should be made to further improve this instrument, by studying and advancing its reliability and by additionally assessing its validity. Specifically, more research should be undertaken to investigate the content validity of this instrument, by assessing if the ped-mTNS is complete in measuring all the aspects of VIPN in terms of relevance and comprehensibility of this instrument, both for patients and assessor. Finally, it should be investigated to which extent an adapted version of the ped-mTNS could be developed that is suitable for assessing VIPN in children under the age of 5. All above-mentioned efforts may result in a more valid and reliable instrument for the assessment of VIPN in children. Meanwhile, using the current version of the ped-mTNS is advocated since it will lead to more uniformity in assessing VIPN in children with cancer, thereby enabling the comparison of study results for this group of patients [37].

In conclusion, the current study showed that the Dutch translated version of the ped-mTNS has good construct validity, whereas reliability appeared insufficient, although patient numbers for reliability testing were low. In order to improve the comparability of results across different studies investigating VIPN in children, further standardization of VIPN assessment is needed. More research aimed at investigating and improving the quality of the ped-mTNS, or in the development of another instrument to assess VIPN, is needed. This will ultimately lead to a robust instrument and more uniformity in evaluating chemotherapy-induced peripheral neuropathy in children with cancer.

Data availability The authors had full control of all primary data and the data that support the findings of this study are available from the corresponding author upon reasonable request.

\section{Compliance with ethical standards}

The Medical Ethics Review Committee of Amsterdam UMC location VUmc determined that the Medical Research Involving Human Subjects Act (WMO) did not apply to this study. Informed consent was obtained from all parents of participating children and from participants themselves in case they were 12 years or older.

Conflict of interest The authors declare that they have no conflicts of interest.

Open Access This article is distributed under the terms of the Creative Commons Attribution 4.0 International License (http:// creativecommons.org/licenses/by/4.0/), which permits unrestricted use, distribution, and reproduction in any medium, provided you give appropriate credit to the original author(s) and the source, provide a link to the Creative Commons license, and indicate if changes were made.

\section{References}

1. Mora E, Smith EM, Donohoe C, Hertz DL (2016) Vincristineinduced peripheral neuropathy in pediatric cancer patients. Am J Cancer Res 6(11):2416-2430

2. Gilchrist LS, Marais L, Tanner L (2014) Comparison of two chemotherapy-induced peripheral neuropathy measurement approaches in children. Support Care Cancer 22(2):359-366

3. Smith EM, Beck SL, Cohen J (2008) The total neuropathy score: a tool for measuring chemotherapy-induced peripheral neuropathy. Oncol Nurs Forum 35(1):96-102

4. Gilchrist LS, Tanner L (2013) The pediatric-modified total neuropathy score: a reliable and valid measure of chemotherapy-induced peripheral neuropathy in children with non-CNS cancers. Support Care Cancer 21(3):847-856 
5. Anghelescu DL, Faughnan LG, Jeha S, Relling MV, Hinds PS, Sandlund JT, Cheng C, Pei D, Hankins G, Pauley JL, Pui CH (2011) Neuropathic pain during treatment for childhood acute lymphoblastic leukemia. Pediatr Blood Cancer 57(7):1147-1153. https://doi.org/10.1002/pbc.23039

6. van de Velde ME, Kaspers GL, Abbink FCH, Wilhelm AJ, Ket JCF, van den Berg MH (2017) Vincristine-induced peripheral neuropathy in children with cancer: a systematic review. Crit Rev Oncol Hematol 114:114-130. https://doi.org/10.1016/j.critrevonc.2017. 04.004

7. Renbarger JL, McCammack KC, Rouse CE, Hall SD (2008) Effect of race on vincristine-associated neurotoxicity in pediatric acute lymphoblastic leukemia patients. Pediatr Blood Cancer 50(4): 769-771. https://doi.org/10.1002/pbc.21435

8. Diouf B, Crews KR, Lew G, Pei D, Cheng C, Bao J, Zheng JJ, Yang W, Fan Y, Wheeler HE, Wing C, Delaney SM, Komatsu M, Paugh SW, McCorkle JR, Lu X, Winick NJ, Carroll WL, Loh ML, Hunger SP, Devidas M, Pui C-H, Dolan ME, Relling MV, Evans WE (2015) Association of an inherited genetic variant with vincristine-related peripheral neuropathy in children with acute lymphoblastic leukemia. JAMA J Am Med Assoc 313(8):815-823

9. Crom WR, De Graaf SS, Synold T, Uges DR, Bloemhof H, Rivera G, Christensen ML, Mahmoud H, Evans WE (1994) Pharmacokinetics of vincristine in children and adolescents with acute lymphocytic leukemia. J Pediatr 125(4):642-649

10. Egbelakin A, Ferguson MJ, MacGill EA, Lehmann AS, Topletz AR, Quinney SK, Li L, McCammack KC, Hall SD, Renbarger JL (2011) Increased risk of vincristine neurotoxicity associated with low CYP3A5 expression genotype in children with acute lymphoblastic leukemia. Pediatr Blood Cancer 56(3):361-367

11. Skiles JL, Chiang C, Li CH, Martin S, Smith EL, Olbara G, Jones DR, Vik TA, Mostert S, Abbink F, Kaspers GJ, Li L, Njuguna F, Sajdyk TJ, Renbarger JL (2018) CYP3A5 genotype and its impact on vincristine pharmacokinetics and development of neuropathy in Kenyan children with cancer. Pediatr Blood Cancer 65(3):e26854. https://doi.org/10.1002/pbc.26854

12. Gohar SF, Marchese V, Comito M (2010) Physician referral frequency for physical therapy in children with acute lymphoblastic leukemia. Pediatr Hematol Oncol 27(3):179-187. https://doi.org/ 10.3109/08880010903580209

13. National Institutes of Health NCI (2010) Common Terminology Criteria for Adverse Events (CTCAE) version $4.03 \mathrm{https} / /$ evs.nci. nih.gov/ftp1/CTCAE/CTCAE_4.03/CTCAE_4.03_2010-06-14 QuickReference_8.5x11.pdf

14. Lavoie Smith EM, Li L, Hutchinson RJ, Ho R, Burnette WB, Wells E, Bridges C, Renbarger J (2013) Measuring vincristine-induced peripheral neuropathy in children with acute lymphoblastic leukemia. Cancer Nurs 36(5):E49-E60. https://doi.org/10.1097/NCC. 0b013e318299ad23

15. Lavoie Smith EM, Li L, Chiang C, Thomas K, Hutchinson RJ, Wells EM, Ho RH, Skiles J, Chakraborty A, Bridges CM, Renbarger J (2015) Patterns and severity of vincristine-induced peripheral neuropathy in children with acute lymphoblastic leukemia. J Peripher Nerv Syst 20(1):37-46

16. Vainionpaa L, Kovala T, Tolonen U, Lanning M (1995) Vincristine therapy for children with acute lymphoblastic leukemia impairs conduction in the entire peripheral nerve. Pediatr Neurol 13(4):314-318

17. Reinders-Messelink HA, Van Weerden TW, Fock JM, Gidding CE, Vingerhoets HM, Schoemaker MM, Goeken LN, Bokkerink JP, Kamps WA (2000) Mild axonal neuropathy of children during treatment for acute lymphoblastic leukaemia. Eur J Paediatr Neurol 4(5):225-233. https://doi.org/10.1053/ejpn.1999.0310

18. Binner M, Ross D, Browner I (2011) Chemotherapy-induced peripheral neuropathy: assessment of oncology nurses' knowledge and practice. Oncol Nurs Forum 38(4):448-454. https://doi.org/ 10.1188/11.ONF.448-454

19. Smith EM, Campbell G, Tofthagen C, Kottschade L, Collins ML, Warton C, Ghosh B, Ronis DL, Mallory GA, Visovsky C (2014) Nursing knowledge, practice patterns, and learning preferences regarding chemotherapy-induced peripheral neuropathy. Oncol Nurs Forum 41(6):669-679. https://doi.org/10.1188/14.ONF.669-679

20. Gilchrist LS, Tanner L, Hooke MC (2009) Measuring chemotherapyinduced peripheral neuropathy in children: development of the PedmTNS and pilot study results. Rehabil Oncol 27(3):7-15

21. DCOG Protocol ALL-11 (2013) Treatment study protocol of the Dutch Childhood Oncology Group for children and adolescents (119 year) with newly diagnosed acute lymphoblastic leukemia. Version 4.0. The Hague, the Netherlands. www.skion.nl

22. The EuroNet-PHL-C1 protocol (2012) First international intergroup study for classical Hodgkin's lymphoma in children and adolescents. www.skion.nl

23. The EuroNet-PHL-C2 protocol (2016) Second international intergroup study for classical Hodgkin's lymphoma in children and adolescents. Version 3.0 www.skion.nl

24. SIOP Wilms (2001) Chemotherapy before and after surgery in treating children with Wilm's tumor www.skion.nl

25. The EpSSG protocol (2012) A protocol for non metastatic rhabdomyosarcoma. Version 1.3 www.skion.nl

26. Bell JA (1984) Semmes-Weinstein monofilament testing for determining cutaneous light touch/deep pressure sensation. The Star 44(2):8-11

27. Medical Research Council of the United Kingdom (1976) Aids to examination of the peripheral nervous system. Memorandum no 45, vol 1976. Her Majesty's Stationary Office, London

28. Jakacki RI, Burger PC, Kocak M, Boyett JM, Goldwein J, Mehta M, Packer RJ, Tarbell NJ, Pollack IF (2015) Outcome and prognostic factors for children with supratentorial primitive neuroectodermal tumors treated with carboplatin during radiotherapy: a report from the Children's Oncology Group. Pediatr Blood Cancer 62(5):776-783

29. Hilz MJ, Axelrod FB, Hermann K, Haertl U, Duetsch M, Neundorfer B (1998) Normative values of vibratory perception in 530 children, juveniles and adults aged 3-79 years. J Neurol Sci 159(2):219-225

30. Nunnally JC, Bernstein IH (1994) Psychometric theory 3rd. McGraw-Hill, New York

31. Hinkle D, Wiersma W, Jurs S (2003) Applied statistics for the behavioral sciences, 5th edn. Houghton Mifflin, Boston

32. McGraw KO, Wong SP (1996) Forming inferences about some intraclass correlation coefficients. Psychol Methods 1(1):30-46

33. Terwee CB, Bot SD, de Boer MR, van der Windt DA, Knol DL, Dekker J, Bouter LM, de Vet HC (2007) Quality criteria were proposed for measurement properties of health status questionnaires. J Clin Epidemiol 60(1):34-42. https://doi.org/10.1016/j.jclinepi.2006.03.012

34. de Vet HC, Mokkink LB, Terwee CB, Hoekstra OS, Knol DL (2013) Clinicians are right not to like Cohen's kappa. BMJ 346:f2125

35. de Vet HC, Terwee CB, Mokkink LB, Knol DL (2011) Measurement in medicine - a practical guide. Cambridge University Press, New York

36. Anthoine E, Moret L, Regnault A, Sebille V, Hardouin JB (2014) Sample size used to validate a scale: a review of publications on newly-developed patient reported outcomes measures. Health Qual Life Outcomes 12:176-186

37. Smolik S, Arland L, Hensley MA, Schissel D, Shepperd B, Thomas K, Rodgers C (2018) Assessment tools for peripheral neuropathy in pediatric oncology: a systematic review from the Children's Oncology Group. J Pediatr Oncol Nurs 35(4):267-275

Publisher's note Springer Nature remains neutral with regard to jurisdictional claims in published maps and institutional affiliations. 